\title{
Solitary Anterior Abdominal Wall Leiomyoma Arisingfrom Tubectomy Scar
}

\author{
K. L. Narasimha Rao ${ }^{1}$, S.S.R.Nagendra Babu, J.Arjun Prakash ${ }^{3}$, \\ G.Sri ram ${ }^{4}$, N.Mohan rao $^{5}$ \\ ${ }^{1}$ Associate professor, Department of General Surgery, GSL Medical College. \\ ${ }^{2}$ Junior resident, Department of General Surgery, GSL Medical College. \\ ${ }^{3}$ Junior resident, Department of General Surgery, GSL Medical College. \\ ${ }^{4}$ Junior resident, Department of General Surgery, GSL Medical College. \\ ${ }^{5}$ Prof \&HOD Department of General Surgery, GSL Medical College
}

\begin{abstract}
:
Background: Anterior abdominal wall fibroid is uncommon and could be a cause of pain and discomfort. Very few cases have been reported in the literature but none from our region.

Case presentation: Hence we present an uncommon case of a 45 year old para $2+2$ who presented with a one year history of a right iliac fossa mass, had surgical removal of the mass and histology report revealed Leiomyoma.

Conclusion: Abdominal wall fibroid is a good differential diagnosis to be considered in any woman of reproductive age with an anterior abdominal mass with previous uterine surgery, including laparoscopic surgeries, which is very rare entity in tubectomy

Introduction

Human leiomyomas are quite common smooth muscle cell tumours that may cause bleeding or a mass effect on adjacent structures. These are one of the most common reasons for hysterectomies in women. These have been thought to be clonal tumours arising from a single myometrial cell. ${ }^{1,2}$ These tumours enlarge during reproductive years and regress later after menopause indicating a role played by estrogen in their genesis and growth. Treatment with agonists of GnRH, which inhibits gonadal function and decrease serum E2, has shown to produce a $40 \%$ to $60 \%$ reduction in the size of a Leiomyoma. The Leiomyoma reverses to its original size if the treatment with GnRH is discontinued. ${ }^{2}$ Treatment with steroids have also been shown to affect the long term results of such tumors. ${ }^{3}$ Deeply situated tumours are likely to be confused with a well differentiated malignant tumour while the superficial lesions are readily recognized as benign.
\end{abstract}

Key words: Leiomyoma, Anterior abdominal wall

\section{Case presentation}

A 45-year old obese multiparous of gravid 2 para 2 (G2P2), perimenopausal lady presented with a mass in the right iliac fossa of one year duration. She had a history of tubectomy 20 years ago. She was not on steroids or hormone replacement therapy. There is no history of gynaecologic interventions. There was no history of seizures, mental retardation, behaviour problems and there were no abnormalities of skin pigmentation

Clinical presentation: On Clinical examination lower abdominal distension noted with $16 \times 10 \mathrm{~cm}$ oval mass at right iliac fossa. The lump was not moving with respiration, not falling forward on knee elbow position. Renal angles are normal and umbilicus midline everted. Visible infra umbilical vertical midline scar present, no hypertrophy or pigmentation. No shifting of dullness, normal bowel sounds, Left supraclavicular fossa normal. Per vaginal and per rectal examination normal

Investigations; Ultrasound abdomen and pelvis showed a large hyper echoic well defined lesion in the posterior wall of uterus suggestive of posterior wall fibroid/ ovarian mass/peritoneal mass. Rest of the visualized visceral organs appears to be normal.

MRI: A large well defined circumscribed lobulated soft tissue mass predominantly solid with cystic areas was noted in the right iliac fossa, the mass measuring $14.4 \times 12 \times 6.3 \mathrm{~cm}$ may be mesenchymal origin.

Intraoperative findings: Laparotomy revealed intra peritoneal minimal fluid collection with a $15 \mathrm{x} 11 \mathrm{x} 8 \mathrm{cms}$ large grayish white well circumscribed solid mass present at right iliac fossa. It was arising from tubectomy scar .Liver, kidney, spleen, ovaries, and uterus and vital organs are normal

Management: Excision of mass was done and specimen sent for Histopathological examination.

Histopathology: The histopathology reported circumscribed tumours made up of long and short intersecting fascicles of smooth muscle cells showing focal area of hyalinization. No nuclear atypia or increased mitotic activity seen. The features are in favour of LEIOMYOMA. 
Follow-up: Postoperatively the patient status and general condition improved and uneventful. After repeated follow ups at weekly intervals for first two weeks, monthly intervals for next 3 months, patient recovered completely.

\section{Discussion}

Leiomyomas are common benign uterine tumours occurring in about $20 \%$ to $30 \%$ of premenopausal women. ${ }^{4}$ The incidence falls sharply in postmenopausal women as it is believed that hormonal changes assist spontaneous resolution of such tumours. Extra uterine leiomyomas are rare events and usually occurs with seeding of myomatous tissues during previous gynaecological surgeries more commonly with laparoscopic procedures. ${ }^{5-7}$ Origin of smooth muscle cell tumours in the anterior abdominal wall from the cells of vessel wall has also been postulated, ${ }^{8}$ especially in those receiving hormone replacement therapy. . The alteration of such cells to Leiomyoma involves somatic mutation and unknown synergistic action of hormones, deranged lipoid metabolism and local growth factors. ${ }^{1,2,4}$ Progesterone and insulin like growth factors have been implicated in Leiomyoma transformation of a smooth muscle cell. Although lipo-leiomyoma arising from anterior abdominal wall without the presence of above mentioned factors have been reported in a case earlier ${ }^{2}$ but isolated Leiomyoma arising de novo in the anterior abdominal wall muscles has not been reported so far.

Moreover, recently it has been found that adiposite may enhance the proliferation of human Leiomyoma cells via TNF- $\alpha$ pro-inflammatory cytokine that is associated a proliferative gene profile in human uterine Leiomyoma cells may play a role in initiation and/or progression of uterine Leiomyoma (HuLM) cells. ${ }^{8}$ The main differential diagnosis of Leiomyoma of the abdominal wall includes well differentiated tumours arising from fat cells, fibrous tissue, and muscle or mesothelial cells. Majority of such tumours are benign. Malignant transformation of smooth muscle tumours have been reported especially in the disseminated variety. 9 The diagnosis of de novo Leiomyoma of the anterior abdominal wall can only be made when there is no history of previous abdominal surgery, open or laparoscopic, because the appearance of leiomyomas are known to occur in scars as old as 30 years. $^{6}$ In cases where a smooth muscle Leiomyoma has been removed previously irrespective of the route, seeding of the scar has been known to give rise to the formation of the tumour later in life.

Parasitic leiomyomas have been reported in the retro- or pre-peritoneum. It has been proposed that the uterine mass becomes adherent to these structures, develops its own blood supply and gradually over the period of time, it loses attachment with the organ of origin, thus developing as a 'parasite' at the new location. ${ }^{10,11}$ This case is unusual as there are neither antecedent gynaecologic complaints nor any history of gynaecologic surgery in the patient. The only surgery she underwent was a tubectomy 20 years prior to her present complaint. A tubectomy operation giving rise to seeding of a Leiomyoma away from the incision site on the anterior abdominal wall is the point to be considered. If it had arisen in the previous scar, it could have been said, for arguments' sake that smooth muscle cells from the fallopian tubes have been implanted in the scar. So far we have not come across any literature to support our hypothesis of seeding of smooth muscle cell from the fallopian tubes.

Every effort should be made to remove the tumour en-block and avoid spillage to prevent the seeding/recurrence of the tumour. In case of a large defect arises after the excision of tumour, synthetic mesh can be used to provide immediate wound cover.

\section{Conclusion}

A Leiomyoma, although commonly seen in uterus or gastrointestinal tract, can develop de novo in areas devoid of myomatous tissues or obvious smooth muscle cells. Whether smooth muscle cells in vessels of anterior abdominal wall react to extraneous hormonal stimulation to form a de novo Leiomyoma is yet to be established. This condition has to be kept in mind while completing the list of differential diagnosis of anterior abdominal wall tumours. However the smooth muscle cells from the fallopian tubes implanted in the scar as seen in the present case is very rare entity.

\section{References}

[1] Porter KB, Tsibris JC, Nicosia SV, Murphy JM, O’Brien WF, Rao PS, et al. Estrogen- induced guinea pig model for uterine leiomyomas: do the ovaries protect? Biol Reprod 1995, Apr; 52(4):824-832.10.1095/biolreprd52.4.824

[2] Lalor PF, Uribe A, Daum GS. De novo growth of a large preperitoneal lipoleiomyoma of the abdominal wall. Gynecol Oncol 2005. May;97(2):719-721.10.1016/j.ygyno.2005.01.050

[3] Stewart EA, Friedman AJ. Steroidal treatment of myomas: Preoperative and long term medical therapy. Semin Reprod Endocrinol 1992;10:344-357.10.1055/s-2007-1018893

[4] Ostrzenski A. Uterine leiomyoma particle growing in an abdominal-wall incision after laparoscopic retrieval. Obstet Gynecol 1997. May;89(5 Pt 2):853-854.10.1016/S0029-7844(97)81428-7

[5] Abdominal Wall after Laparoscopic Myectomy. Fertil Streil. 2008 Apr 12. Epub Ahead of Print.

[6] Kumar S, Sharma JB, Verma D, Gupta P, Roy KK, Malhotra N. Disseminated peritoneal leiomyomatosis: an unusual complication of laparoscopic myomectomy. Arch Gynecol Obstet 2008. Jul;278(1):93-95.10.1007/s00404-007-0536-9 
[7] Schindle M, Birner P, Losch A, Breitenecker G, Joura EA. Preperitoneal Lipoleiomyoma of the Abdominal wall in a postmenopausal woman. Maturitas 2001;30:189.10.1016/S0378-5122(01)00271-7

[8] Nair S, Al-Hendy A. Adipocytes enhance the proliferation of human leiomyoma cells via TNF-á Proinflammatory cytokine. Reprod Sci 2011;8(12):1186- 192.10.1177/ 1933719111408111

[9] Wada Hiraike O, Yamamoto N, Osuga Y, Yano T, Kozuma S, Taketani Y, Aberrant implantation and growth of uterine leiomyoma in the abdominal wall after laparoscopically assisted myomectomy. Fertil.Sterilit. 2009; 92(5):1747.e13-1747.e15.

[10] Yamaguchi T, Imamura Y, Yamamoto T, Fukuda M. Leiomyomatosis peritonealis disseminata with malignant change in a man. Pathol Int 2003. Mar;53(3):179-185.10.1046/j.1440-1827.2003.01452.x11. Berhan Y, Isehak A, Legesso S, Tsegaye B. Pseudo-Meig’s syndrome: parasitic leiomyoma with ascites in a 52-year old lady. Ethiop Med J 2003. Oct;41(4):363-366.

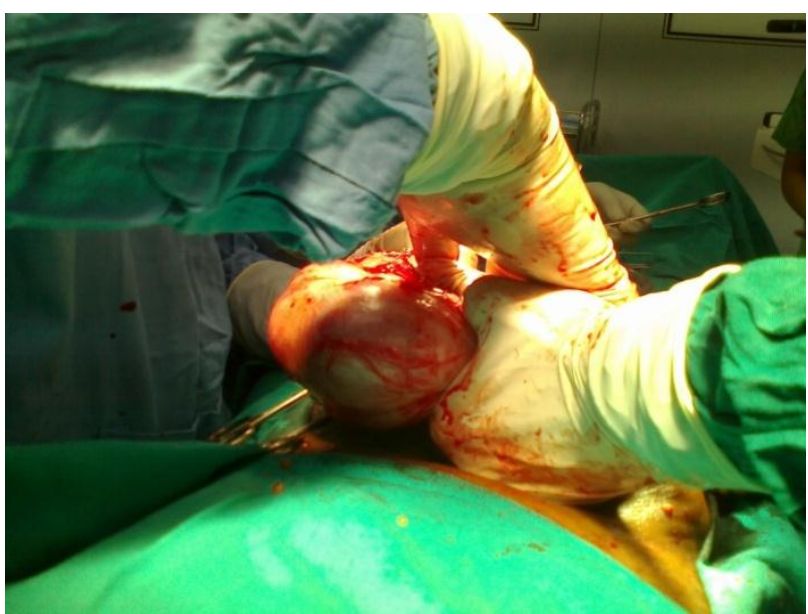

Figure 1 intra operative excision of leiomyoma

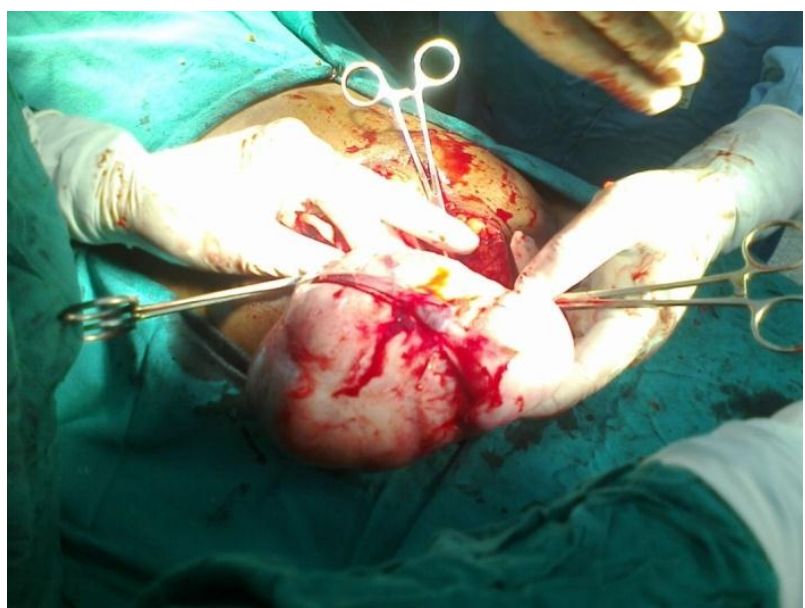

Figure 2 intraoperative excision of leiomyoma from scar tissue

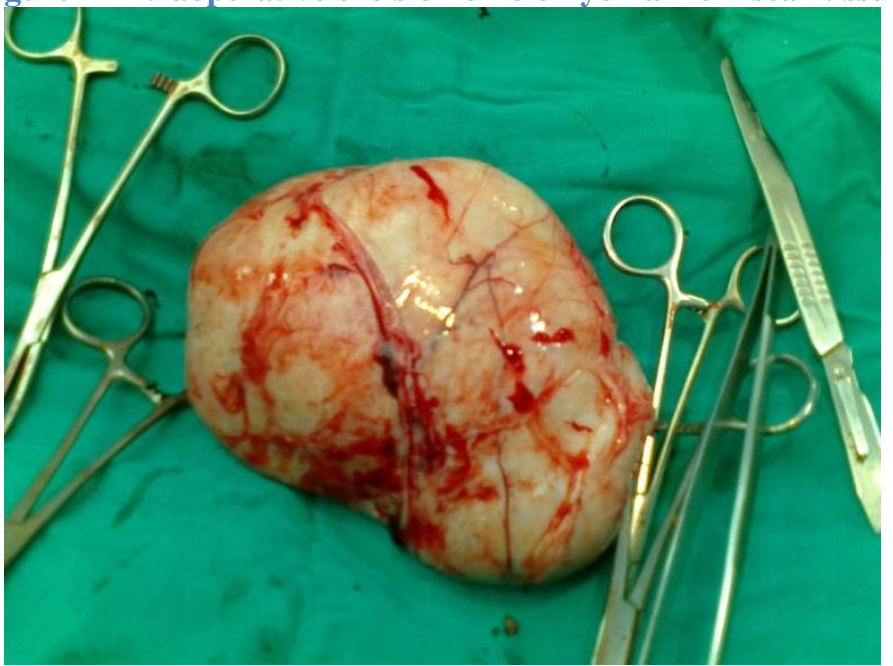

Figure 3 excised specimen 